\title{
Macromolecule Permeability in Rodent Intestine following Thermal Injury and Lipopolysaccharide Challenge
}

\author{
Peirong Yu ${ }^{1,2}$ and Edward A. Carter ${ }^{2,3}$ \\ ${ }^{1}$ Rhode Island Hospital, Brown University School of Medicine, SWP-511, 593 Eddy Street, Providence, RI 02903, USA \\ ${ }^{2}$ Departments of Surgery and Pediatrics, Massachusetts General Hospital and Harvard Medical School, Charlestown, MA 02114, USA \\ ${ }^{3}$ Massachusetts General Hospital, Pediatric Gastrointestinal Unit, 114 16th Street (114-3503), Charlestown, MA 02129-4004, USA
}

Correspondence should be addressed to Edward A. Carter; carter.ea@gmail.com

Received 30 April 2013; Accepted 30 May 2013

Academic Editors: K. Shirasuna, A. Tse, and D. Xiao

Copyright (C) 2013 P. Yu and E. A. Carter. This is an open access article distributed under the Creative Commons Attribution License, which permits unrestricted use, distribution, and reproduction in any medium, provided the original work is properly cited.

\begin{abstract}
The barrier function of the intestinal mucosa may be lost during stress such as severe trauma and sepsis. The present study utilized a multicannulated (jugular vein, proximal jejunum, thoracic duct, and portal vein) rat model of burn (30\% body surface area (TBSA)) and endotoxemia (E. coli lipopolysaccharide (LPS) infused via the jugular cannula) to investigate in vivo barrier function to macromolecules with different sizes and the route for their transport (horse radish peroxidase (HRP) and 14C-polyethylene glycol (PEG)-4,000 infused via jejunal cannula). In burn rats, mucosa uptakes of HRP and PEG increased $3 \mathrm{~h}$ after their intraluminal infusion compared to the controls. Studies with intravenous ${ }^{111} \mathrm{In}-\mathrm{IgG}$ infusion showed that its recovery in small intestine was decreased after burn and LPS infusion, indicating that blood perfusion to intestine was compromised. The present study suggests that (1) burn and endotoxemia increase intestinal permeability to macromolecules; (2) the portal blood may be the major route of transport for molecules up to sizes of 4,000 during burn but not endotoxemia; and (3) intestinal hypoperfusion could be one of the factors that contribute to increased gut permeability in severe burn trauma and sepsis.
\end{abstract}

\section{Introduction}

In addition to its role in nutrient absorption, the intestinal mucosa functions as a major barrier to the passage of infectious and/or potentially toxic macromolecules present in the gut lumen. However, this barrier function may be lost under certain circumstances, such as hemorrhagic shock, severe trauma, and sepsis, leading to the subsequent development of systemic sepsis and multiple organ failure syndrome $[1,2]$. It has been demonstrated in animal model in vivo that endotoxin and thermal injury could induce bacterial translocation from gut lumen to mesenteric lymph nodes, liver, or spleen $[3,4]$. We have previously shown that, using the everted intestinal sac model in vitro, $20 \%$ body surface area (BSA) thermal injury enhances the small intestinal permeability to a variety of macromolecules such as horse radish peroxidase and polyethylene glycol [5]. We have also demonstrated that there is a markedly diminished in vivo incorporation of tritiated thymidine into the small intestinal mucosa for a transient period after these injuries [6].

In the present study, we established a multiple cannulated whole animal model to investigate the intestinal barrier function during stress and the possible routes of macromolecule passage after they penetrate the intestinal mucosa.

\section{Materials and Methods}

2.1. Animals. All experiments were carried out using 250 $450 \mathrm{~g}$ albino Charles River strain male rats maintained before study on a standard laboratory diet. Male rats were used because of the larger size at a young age, making the multiple cannulation possible. Animals were anesthetized using halothane inhalation after an overnight fast. The flow rate of halothane was well controlled using a flow rate controller to obtain a satisfactory degree of anesthesia for surgery without respiratory depression. The right jugular vein was cannulated 
first. With a midline incision of the abdomen, the proximal jejunum, $3 \mathrm{~cm}$ distal to the ligament of Treitz, was then cannulated using a PE-50 tubing. The subdiaphrenic portion of the thoracic duct was exposed by blunt dissection with extreme cautions. This is better done under a microscope. Extreme care has also to be taken not to penetrate the diaphragm. The thoracic duct was then cannulated with a PE90 tubing just beneath the diaphragm pointing to distal, fixed with two 6-0 silk sutures. The portal vein was cannulated with a PE-50 tubing and sealed with surgical glue so that the portal blood flow was preserved. The abdominal wall was closed routinely. After surgery, animals were placed in Bollman cages with minimal restriction. They were then placed in a well-ventilated incubator with a constant temperature of $33^{\circ} \mathrm{C}$ for up to two hours to help the animals recover from anesthesia. The recovery was monitored by judging their overall activity and measuring body temperature with a rectal probe until the body temperature returned to normal levels. Animals were then allowed to stabilize overnight with free access to food and water. Studies were carried out the next day.

All animal studies described here were approved and performed in strict accordance to the guidelines established by the Massachusetts General Hospital Animal Care and Use Committee.

2.2. Thermal Injury and LPS Administration. Cutaneous thermal injuries were carried out by the methods of Walker and Mason [7] as modified [8]. Under ether anesthesia, the backs of rats were shaved and placed in a mold exposing approximately 30\% TBSA (Total Body Surface Area calculated as described previously [8]). The backs of rats were then placed on top of a boiling water bath for 10 seconds. The rats were then given an intraperitoneal injection of normal saline (15 $\mathrm{mL} / \mathrm{kg}$ body weight) for resuscitation. Rats in control groups were shammed with the same procedure except burn. The burn injury used in these studies produces a third degree full thickness burn. Pain is not considered to be associated with this injury as it has been shown by Osgood et al. [9], that plasma beta-endorphin levels and tail flick latency increase in this model, and Ward et al. [10] have shown that nerve endings in the skin are destroyed in this model.

E. coli lipopolysaccharide (LPS) 0111:B4 was dissolved in normal saline at a final concentration of $0.2 \mathrm{mg} / \mathrm{mL}$. Doses of $0.1 \mathrm{mg} / \mathrm{kg}$ and $0.3 \mathrm{mg} / \mathrm{kg}$ body weight were injected into the jugular vein via the jugular cannula. The same volume of saline was injected in the control animals.

2.3. Macromolecule Infusion. Horse radish peroxidase (HRP), $20 \mathrm{mg} / \mathrm{kg}$ body weight, was dissolved in $0.1 \mathrm{M}$ phosphate buffer, $\mathrm{pH} 6.0$, at a final concentration of $2 \mathrm{mg} / \mathrm{mL}$. To this solution, $20 \mu \mathrm{Ci} / \mathrm{kg}$ body weight of ${ }^{14} \mathrm{C}$-labeled polyethylene glycol (PEG)-4,000 was added. Three hours after LPS challenge or six hours after burn, the HRP/PEG solution was introduced into the jejunum via the jejunal cannula in a fiveminute duration. Infusion of normal saline to the jejunum was started thirty minutes following the HRP/PEG infusion. A total volume of $5 \mathrm{~mL}$ was constantly infused intraluminally over a period of 150 minutes (until the end of study), using a Harvard pump, to maintain sufficient lymph flow.
2.4. Collection of Lymph and Blood Samples. Following intraluminal administration of HRP/PEG, the entire lymph efflux was collected in ice-cold microcentrifuge tubes at time intervals of every 30 minutes for 3 hours. For each time interval, the volume was recorded and then the samples were stored at $-20^{\circ} \mathrm{C}$ until assay.

Samples of $0.6 \mathrm{~mL}$ of portal blood were taken from the portal vein cannula at time intervals of 30 minutes. One $\mathrm{mL}$ of normal saline was replaced immediately after the blood withdraw. Blood samples were collected in heparinized test tubes and centrifuged at 1,000 g for 15 minutes. The plasma was obtained and stored at $-20^{\circ} \mathrm{C}$ until assay. The hematocrit was recorded each time.

By the end of study, rats were sacrificed with a lethal dose of intravenous pentobarbital. The entire small intestine was removed and the lumen was washed with $300 \mathrm{~mL}$ of phosphate buffer until the effluent was clear. The intestine was cut open longitudinally. The mucosa was scraped, weighed, and homogenized in $0.5 \%$ hexadecyltrimethylammonium bromide (HTAB) in $50 \mathrm{mM}$ phosphate buffer, $\mathrm{pH}$ 6.0, in a volume of 10 times the wet weight of mucosa. Following centrifugation at $30,000 \times \mathrm{g}$ for 15 minutes, the supernatant was collected and kept at $-20^{\circ} \mathrm{C}$ until assay.

2.5. Measurements of HRP and ${ }^{14} C-P E G-4,000$. Quantitative assay of HRP was carried out as described by Warshaw et al. [11]. Briefly, a $0.1 \mathrm{~mL}$ aliquot of sample was added to 2.9 $\mathrm{mL}$ of reaction mixture containing $0.003 \% \mathrm{H}_{2} \mathrm{O}_{2}, 1 \%$ bovine serum alburnin in $0.1 \mathrm{M}$ phosphate buffer, $\mathrm{pH} 6.0$, and 0.025 $\mathrm{mL}$ of O-dianisidine.diHCI $(10 \mathrm{mg} / \mathrm{mL})$. Using a Gilford Spectrophotometer, the rate of increase in optical density at $460 \mathrm{~nm}$ was determined. A standard curve relating enzyme activity to enzyme protein concentration was subsequently used to determine the concentration of HRP protein in samples.

The radioactivity of ${ }^{14} \mathrm{C}$-PEG-4,000 in samples was measured using the Analytic 81 liquid scintillation counter (Tracer Analytic, Des Plaines, IL).

2.6. Drugs and Chemicals. E. coli lipopolysaccharide (LPS) 0111:B4 was purchased from List Biological Laboratories Inc. (Campbell, CA); ${ }^{14}$ C-labeled polyethylene glycol (PEG)4,000 and ${ }^{111}$ In-IgG were purchased from DuPont/New England Nuclear Research Products (Boston, MA). Horse radish peroxidase (HRP) and other drugs were purchased from Sigma Chemical (St. Louis, MO).

2.7. Statistical Analysis. Data were expressed as mean \pm SE. Unpaired student $t$-test and one- and two-factorial repeated analysis of variances (ANOVA) were used for statistical analysis. $P<0.05$ was considered significant.

\section{Results}

\subsection{Burn Group}

3.1.1. Mucosal Level (Figure 1). Three hours after HRP/PEG infusion, average uptake of HRP by intestinal mucosa in rats receiving thermal injury was $2.93 \pm 0.69 \%$ (mean \pm SEM, 


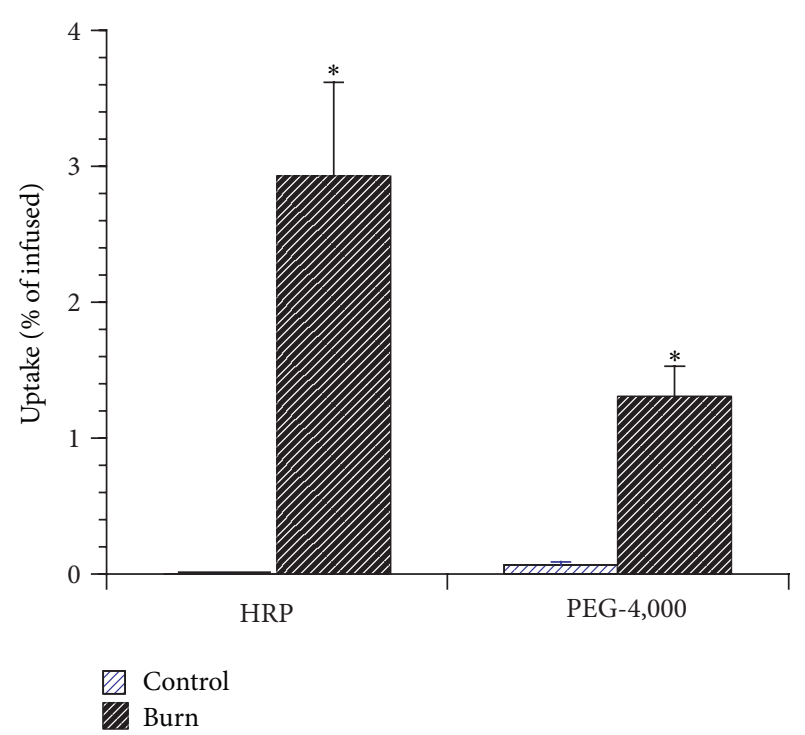

FIgURE 1: Total three-hour uptake of HRP and PEG-4,000 by intestinal mucosa in rats receiving either $30 \%$ BSA burn or sham. Data are expressed as percent from the total amount of infused. Values are mean \pm SEM from 6 animals in each group. ${ }^{*}$ indicates $P<0.001$.

$n=6)$. It was undetectable in all the 6 control animals. The uptake of ${ }^{14} \mathrm{C}$-PEG-4,000 by the gut mucosa was 1.31 $\pm 0.22 \%$ in animals receiving thermal injury compared to $0.07 \pm 0.002 \%$ in control animals $(P<0.001, n=6)$. The mucosal uptake of both macromolecules was higher in the jejunum than in the ileum in burned rats whereas they were not different in control animals. HRP uptake in burned rats was $2.17 \pm 0.76 \%$ and $0.75 \pm 0.36 \%(P<0.05)$ in jejunum and ileum, respectively. PEG-4,000 uptake was $0.92 \pm 0.24 \%$ and $0.4 \pm 0.15 \%$, respectively.

3.1.2. Lymph Level. In this group, four animals received $30 \%$ TBSA burn and the other four sham. The total lymph flow in the three-hour period was $2.5 \pm 0.3 \mathrm{~mL}(n=8)$. There were no differences between the burned and control animals $(2.65 \pm 0.11 \mathrm{~mL}$ and $2.43 \pm 0.2 \mathrm{~mL}$, resp.). The lymph flow rate varied little when intraluminal perfusion of normal saline was maintained constantly.

The total three-hour absorption of HRP and PEG-4,000 increased 4.1- and 3.3-fold, respectively, in burned rats compared to control (Figure 2). Increased absorption of HRP (Figure 3) in burned rats occurred earlier than that of PEG4,000 (Figure 4). The absorption in control rats remained low for both HRP and PEG-4,000 over the $3 \mathrm{~h}$ period.

3.1.3. Portal Level. Portal blood samples were obtained from four control and four burned rats via portal vein cannulas. To ensure that animals were stable over the time period of study, hematocrit was measured for each blood sample. The hematocrits remained stable during the 3 hour period, being typically $45-50 \%$ with no differences between burn and control rats. No gross hemolysis occurred. Plasma concentrations of HRP and ${ }^{14}$ C-PEG-4,000 were measured before infusion and at $30 \mathrm{~min}$ intervals after infusion. Low activities of HRP were

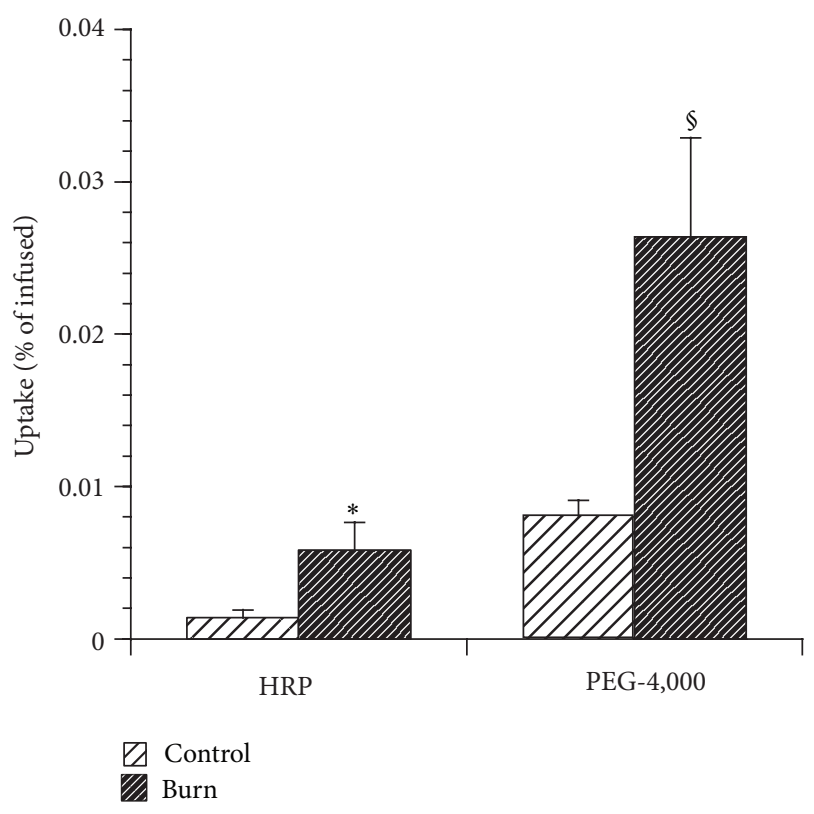

FIgURE 2: Total three-hour absorption of HRP and PEG-4,000 to lymph in burned rats compared to control animals. Values are mean \pm SEM from 4 animals in each group. ${ }^{*}$ indicates $P<0.05$. ${ }^{\S}$ indicates $P<0.01$.

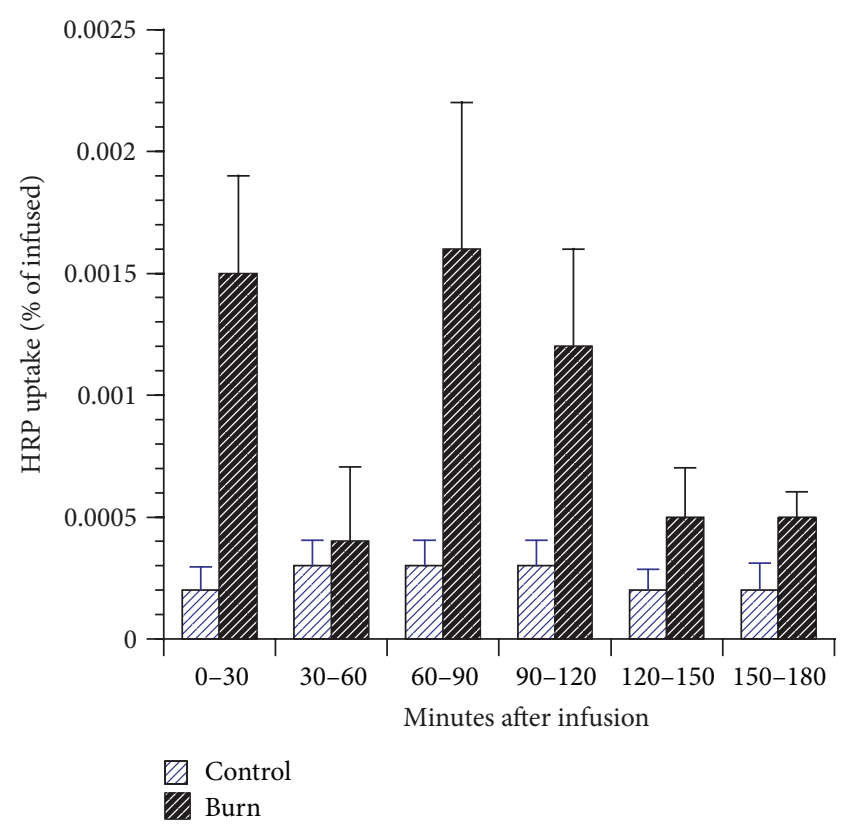

FIGURE 3: Time course of HRP absorption to lymph in rats receiving $30 \%$ BSA burn or sham. Values are mean \pm SEM from 4 animals.

detected in portal blood before HRP/PEG infusion $(0.23 \pm$ $0.07 \mu \mathrm{g} / \mathrm{mL}$ and $0.19 \pm 0.05 \mu \mathrm{g} / \mathrm{mL}$ plasma in burned and control animals, resp., $P>0.5$ ). There were no increases of HRP activity in portal blood samples from either control or burned rats, however, after infusion. Activities of ${ }^{14} \mathrm{C}-\mathrm{PEG}-4,000$ were detected in portal blood as early as $30 \mathrm{~min}$ after infusion in both groups of animals (Figure 5). PEG-4,000 concentration in portal blood increased steadily over the $3 \mathrm{~h}$ period in 


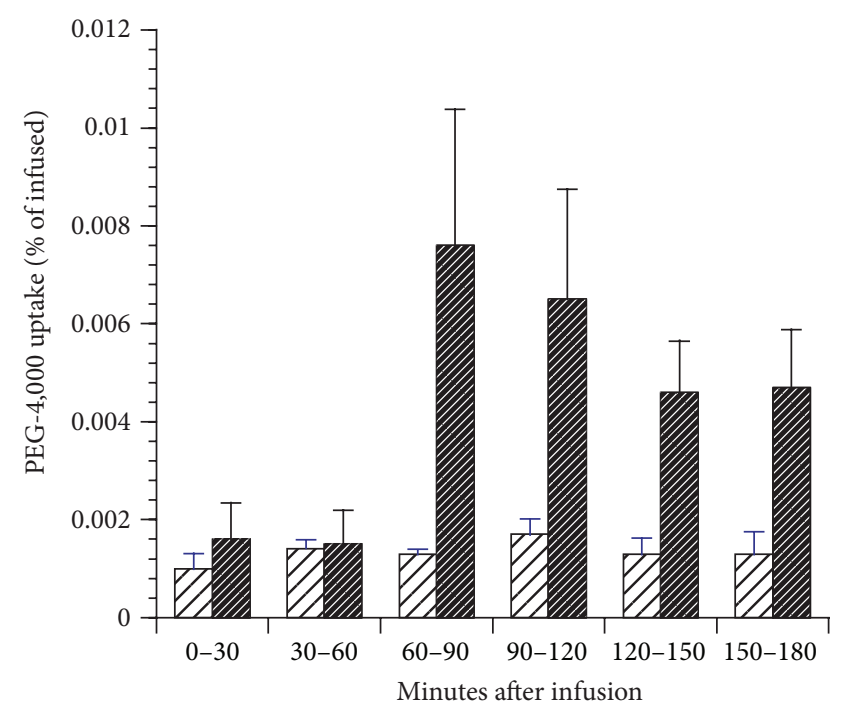

$\bigotimes$ Control

FIgURE 4: Time course of ${ }^{14} \mathrm{C}-\mathrm{PEG}-4,000$ absorption to lymph in rats receiving $30 \%$ BSA burn or sham. Values are mean \pm SEM from 4 animals.

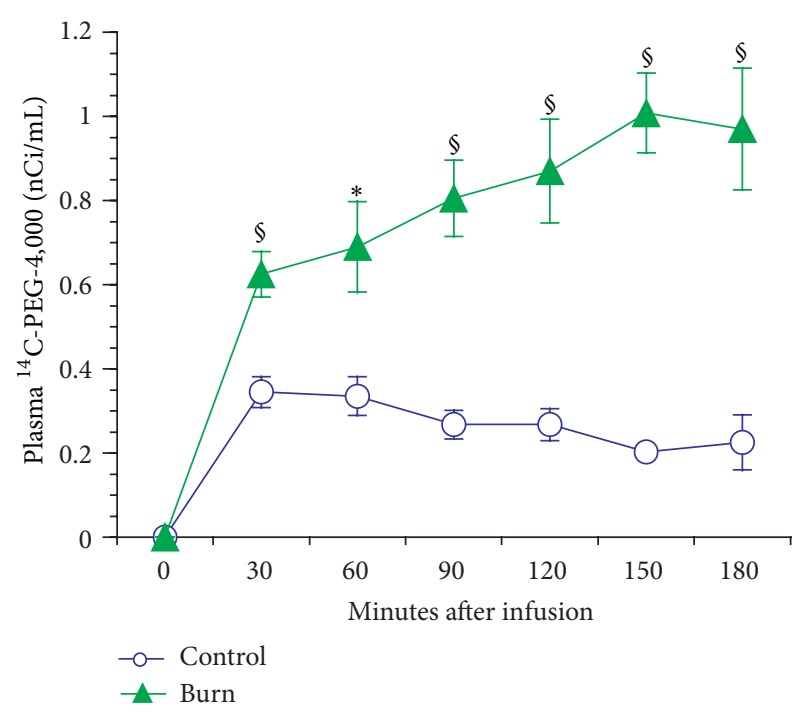

FIGURE 5: Plasma concentrations of ${ }^{14} \mathrm{C}-\mathrm{PEG}-4,000$ in portal blood after intraluminal infusion in rats receiving $30 \%$ BSA burn or sham. Values are mean \pm SEM from 4 animals in each group. The overall PEG-4,000 concentration over time is significantly higher in burned rats than in control $(P<0.001$, ANOVA two-factorial repeated measurements) ${ }^{*}$ indicates $P<0.05$; ${ }^{\S}$ indicates $P<0.01$ (ANOVA, one-factorial measurements).

rats receiving 30\% BSA burn whereas it gradually decreased after $30 \mathrm{~min}$ in control animals ( $P=0.001$, ANOVA).

\subsection{PLS Group}

3.2.1. Mucosal Level. Three groups of rats, 6 each, were included in this study. The control group received intravenous

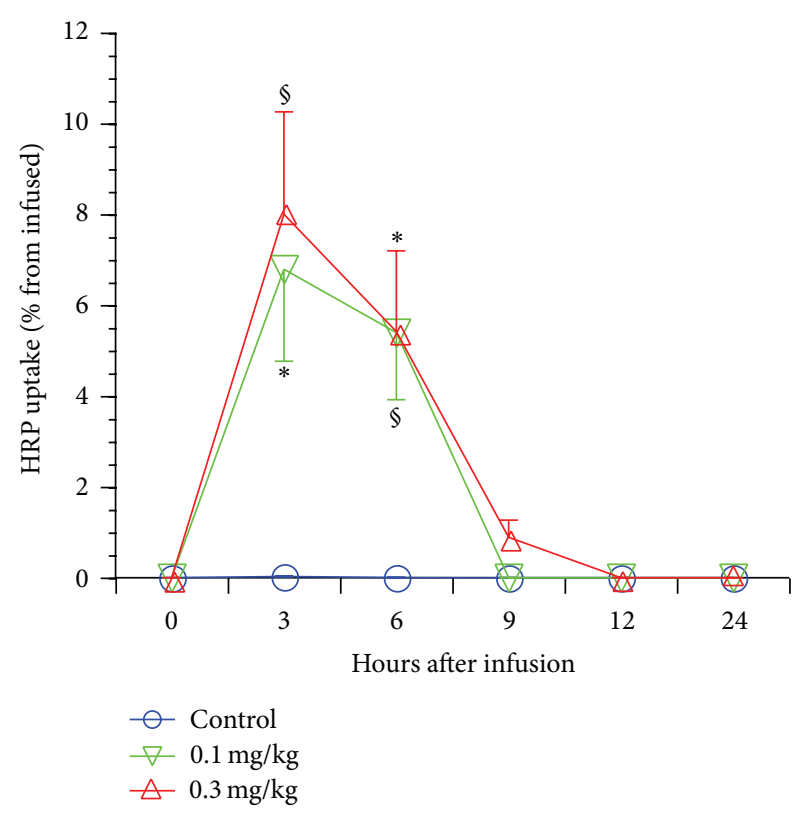

FIgURE 6: HRP uptake by intestinal mucosa 3 to 24 hours after infusion in rats receiving intravenous LPS at 0.1 and $0.3 \mathrm{mg} / \mathrm{kg}$ and normal saline (control). Values are mean \pm SEM from 6 rats in each group. ${ }^{*}$ indicates $P<0.05$; ${ }^{\circledR}$ indicates $P<0.01$ compared to control (ANOVA, one-factorial measurement).

infusion of normal saline. Another group received an LPS dose of $0.1 \mathrm{mg} / \mathrm{kg}$ body weight intravenously. The third group received an intravenous LPS dose of $0.3 \mathrm{mg} / \mathrm{kg}$ body weight. $\mathrm{HRP} /{ }^{14} \mathrm{C}-\mathrm{PEG}-4,000$ infusion started $3 \mathrm{~h}$ after LPS challenge. Intestinal mucosa was obtained before and 3, 6, 9, 12, and 24 hours after intraluminal infusion. Total HRP and ${ }^{14} \mathrm{C}$ PEG-4,000 uptake by mucosa was shown in Figures 6 and 7 , respectively. There was a dramatic increase of uptake at $3 \mathrm{~h}$ for both macromolecules, which returned to control level 9 hours after infusion. The uptakes were slightly higher in rats receiving the higher dose of LPS although they were not statistically significant. In a similar way to that in the burn group, the uptake was higher in jejunum than in ileum in rats receiving $0.3 \mathrm{mg} / \mathrm{kg}$ of LPS. HRP uptake was 2.2 -fold and PEG-4,000 uptake was 3.2-fold higher in jejunal than in ileal mucosa. However, in rats receiving the lower dose of LPS, the uptake of both macromolecules was slightly higher (1.5-fold) in ileum than in jejunum.

3.2.2. Lymph Level. Uptake of HRP and PEG-4,000 to lymph was studied 3, 6, 9, 12, and 24 hours after their infusion in rats receiving either $0.3 \mathrm{mg} / \mathrm{kg}$ body weight of LPS or the same volumes of normal saline (control). Lymph uptake for both macromolecules in the LPS group increased significantly at $3 \mathrm{~h}$ (Figure 8), slightly at $6 \mathrm{~h}$, and unmeasurabl after $9 \mathrm{~h}$.

3.2.3. Portal Level. HRP and ${ }^{14} \mathrm{C}-\mathrm{PEG}-4,000$ activities in portal blood were measured at $30 \mathrm{~min}$ intervals after their intraluminal infusion. A total of 8 animals were studied in this group, 4 receiving $0.3 \mathrm{mg} / \mathrm{kg}$ body weight of LPS and 4 normal saline (control). Low activities of HRP were measurable in portal blood even before HRP infusion and there were 


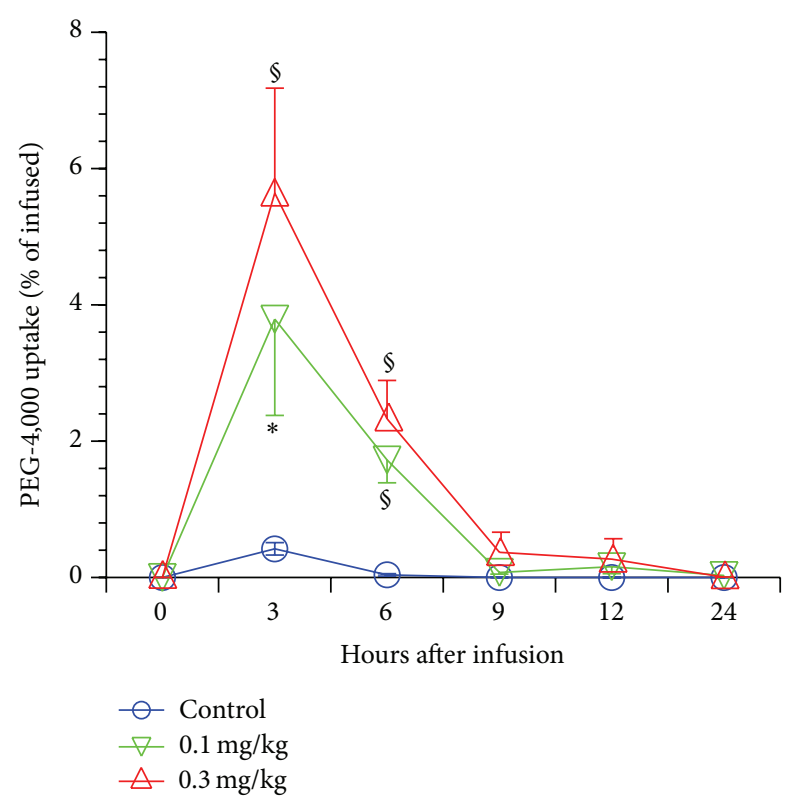

Figure 7: Absorption of ${ }^{14} \mathrm{C}-\mathrm{PEG}-4,000$ by intestinal mucosa 3 to 24 hours after infusion in rats receiving intravenous LPS at 0.1 and $0.3 \mathrm{mg} / \mathrm{kg}$ and normal saline (control). Values are mean $\pm \mathrm{SEM}$ from 6 rats in each group. ${ }^{*}$ indicates $P<0.05$; ${ }^{\circledR}$ indicates $P<0.01$ compared to control (ANOVA, one-factorial measurement).

no increases of HRP activity after infusion in either group. Low activities of ${ }^{14} \mathrm{C}$-PEG-4,000 were detected 30 min after infusion in both groups and decreased steadily afterwards (Figure 9). No significant differences of ${ }^{14} \mathrm{C}$ - PEG-4,000 in portal blood were found between the LPS and control groups.

3.3. Intestinal Microcirculation. To investigate if intestinal microcirculation changes or capillary-to-gut lumen leakage occurs after burn and LPS challenge, In-111-labeled IgG (molecular weight of 160,000) was infused via the jugular vein 6 hours after burn (or sham) and 3 hours after LPS $(0.3 \mathrm{mg} / \mathrm{kg}$ body weight) challenge (or normal saline). Twenty minutes after ${ }^{111} \mathrm{In}-\mathrm{IgG}$ infusion, animals were sacrificed. The entire small intestine (from the ligament of Treitz to ileocecal valve) was removed and cut to two segments of equal length, defined as jejunum and ileum. Intestinal fluid from each segment was collected. The intestinal lumen was washed with $10 \mathrm{~mL}$ normal saline (until being clear) and the washouts were pooled. The total radioactivity in samples from gut lumen was then determined using a $g$ counter. The intestinal tissue was homogenized and their radioactivity was also measured.

Total ${ }^{111}$ In-IgG radioactivity in the entire intestine (tissue + lumen) was lower $(P<0.05)$ in rats receiving burn or LPS administration than in control 7 animals, being $16.6 \pm$ $0.6 \mathrm{nCi}$ in the burn group and $14.4 \pm 0.9 \mathrm{nCi}$ in the LPS group compared to $22.55 \pm 3 \mathrm{nCi}$ in control animals. The activity in gut lumen was typically $4-6 \%$ of that in tissue and its changes after burn and LPS are parallel to those in intestinal tissue, therefore the tissue and lumen activities are combined. The decrease of radioactivity after burn and LPS was more profound in the jejunum than in the ileum (Figure 10).

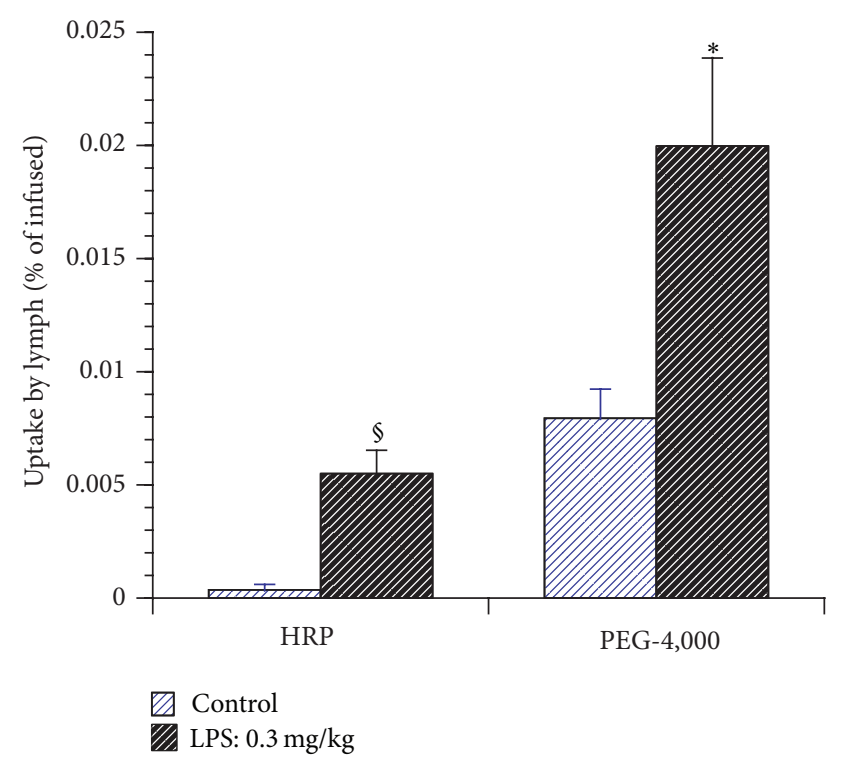

Figure 8: Absorption of HRP and ${ }^{14} \mathrm{C}-\mathrm{PEG}-4,000$ to lymph in rats receiving $0.3 \mathrm{mg} / \mathrm{kg}$ body weight of LPS versus control. Values are mean \pm SEM from 6 rats in each group. ${ }^{*}$ indicates $P<0.05$; ${ }^{s}$ indicates $P<0.01$ compared to control (ANOVA, one-factorial measurement).

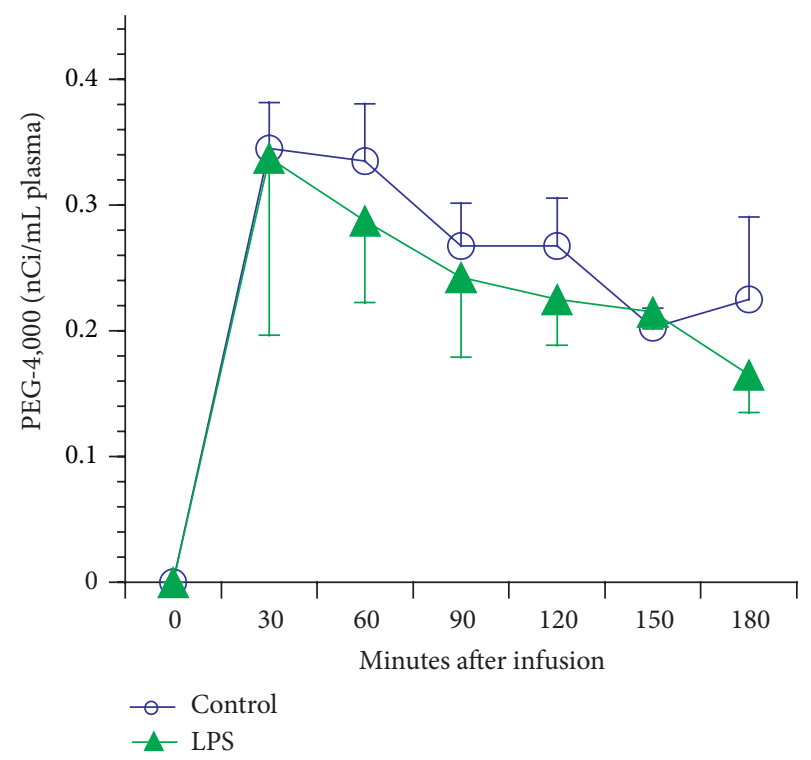

FIGURE 9: Plasma concentration of ${ }^{14} \mathrm{C}-\mathrm{PEG}-4,000$ in portal blood in rats receiving $0.3 \mathrm{mg} / \mathrm{kg}$ body weight of LPS versus control. Values are mean \pm SEM from 4 rats in each group. No significant differences were detected in the two groups.

\section{Discussion}

Nosocomial infections frequently occur in hospitalized patients and may be life threatening if the patient is immunocompromised because of a systemic sepsis or severe trauma [12]. Much attention has been focused on exogenous microorganisms colonizing the skin, upper respiratory tract, or urinary tract of the patient. However, accumulating clinical 


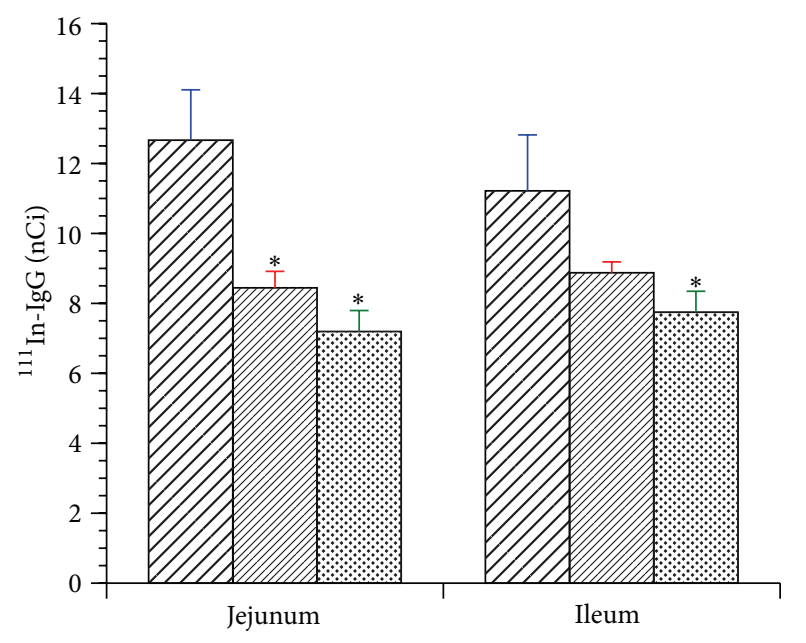

$\square$ Control
$\square$ Burn
LPS

FIGURE 10: Radioactivity of ${ }^{111} \mathrm{In}-\mathrm{IgG}$ recovered in the jejunum and ileum $20 \mathrm{~min}$ after its intravenous infusion in rats receiving $0.3 \mathrm{mg} / \mathrm{kg}$ body weight of LPS versus control. Values are mean \pm SEM of 4 animals in each group. ${ }^{*}$ indicates $P<0.05$ ( $t$-test).

and epidemiological evidence suggests that gut failure is associated with loss of barrier function to bacteria and/or to bacterial endotoxin $[2,12]$. It has been stated that the gut plays a key role in invasive sepsis in surgical patients $[13,14]$. Deitch et al. have demonstrated, using mice as animal model, that enhanced bacterial translocation occurred after endotoxin administration and thermal injury $[2,3]$. Increased gut permeability has also been shown in healthy human volunteers receiving a single dose of endotoxin and in burn patients complicated with infection, in which increased absorption of lactulose, a relatively small molecule with molecular weight of 342 daltons, has been observed $[15,16]$.

The present study showed that both a nonlethal single dose of LPS and 30\% TBSA cutaneous thermal injury promoted the mucosal uptake of macromolecules with molecular weight of 40,000 and 4,000 in rats. A higher dose of LPS tended to cause greater uptake of PEG-4,000 although it was statistically insignificant. In the burn and high LPS groups, the mucosal uptake was higher in the jejunum than in the ileum. These findings are, however, different from those from in vitro studies in which the ileum seemed to play a major role [5]. Methodological differences might explain these variations since the physiological or pathophysiological changes in in vivo studies are much more complex than in vitro studies.

The mechanism(s) of mucosal permeation to macromolecules are still unclear. Unlike physical damage to the gut mucosa, for example, intraluminal ethanol administration, HRP was reported to be absorbed by pinocytosis [11]. Histology study revealed no evidence of ulceration or breaks in the mucosa after thermal injury [16]. Thus, the macromolecule permeation seems to be an active process rather than a passive diffusion under these circumstances. The present study suggests that intestinal hypoperfusion might be one of the factors that contribute to increased gut permeability during stress. Data from the ${ }^{111} \mathrm{In}-\mathrm{IgG}$ experiments showed that less ${ }^{111} \mathrm{In}-\mathrm{IgG}$ was recovered in the small intestine in rats receiving LPS administration and thermal injury than in control animals, indicating that hypoperfusion to the intestine occurred during stress. Moreover, this hypoperfusion was more profound in the jejunum than in the ileum, which correlates well to the greater increase of macromolecule uptake by the jejunum than by the ileum. The present study, however, did not answer the question of how hypoperfusion affects gut permeability. Other factors may play important role in mediating increased intestinal permeability, such as the synthesis and release of various inflammatory mediators after endotoxemia [17]. Among them, platelet-activating factor (PAF) has been considered as a major mediator of septic shock [18]. It has been demonstrated that intravascular infusion of PAF increases vascular permeability and causes hypotension and that specific PAF antagonists are able to reverse endotoxic shock and gastrointestinal damage [1921]. In our preliminary study, we found that a specific PAF antagonist SRI 63-441 was unable to prevent LPS-induced macromolecule uptake by rat intestinal mucosa (data not shown). This finding is in agreement with that of Deitch in which endotoxin-induced bacterial translocation in mice was not prevented by SRI 63-441 [4]. These findings suggest that the effect of endotoxin, at the present type and dose used, may not be primarily mediated by PAF.

The route by which HRP and PEG were absorbed into the body after the mucosal uptake was also investigated in the present study. It showed that $30 \%$ BSA burn and $0.3 \mathrm{mg} / \mathrm{kg}$ body weight of LPS increased HRP and PEG-4,000 absorption to lymph to a similar extent. However, no increases of HRP concentration in portal blood were observed in either group, suggesting that HRP is not transported primarily via portal vein probably because of its large size $(40,000$ compared to 4,000 of PEG). The present study also showed that plasma PEG-4,000 concentrations in portal vein increased considerably after $30 \%$ TBSA burn over control animals whereas no differences were observed in rats receiving LPS infusion and control. The plasma PEG-4,000 concentrations in portal blood were similar to that in lymph (data not shown) but the portal blood flow is 500-650 times the lymph flow [22]. These findings suggest that, in addition to lymphatic transport, the portal blood is another important route or the major route of macromolecule absorption during thermal injury but not during endotoxemia.

In conclusion, the present in vivo study, using a multiple cannulated animal model, provides direct evidence that (1) nonlethal thermal injury and intravenous administration of LPS increase intestinal permeability to macromolecules; (2) the portal blood may be the major route of transport for macromolecules up to 4,000 molecular weight during thermal injury but not endotoxemia; and (3) intestinal hypoperfusion during such stress might be one of the factors that contribute to this intestinal change.

\section{References}

[1] J. R. Border, J. Hassett, J. LaDuca et al., "The gut origin septic states in Blunt multiple trauma (ISS $=40$ ) in the ICU," Annals of Surgery, vol. 206, no. 4, pp. 427-448, 1987. 
[2] C. J. Carrico, J. L. Meakins, and J. C. Marshall, "Multiple-organfailure syndrome," Archives of Surgery, vol. 121, no. 2, pp. 196208,1986

[3] K. Maejima, E. A. Deitch, and R. D. Berg, "Bacterial translocation from the gastrointestinal tracts of rats receiving thermal injury," Infection and Immunity, vol. 43, no. 1, pp. 6-10, 1984.

[4] E. A. Deitch, L. Ma, W. J. Ma et al., "Inhibition of endotoxininduced bacterial translocation in mice," Journal of Clinical Investigation, vol. 84, no. 1, pp. 36-42, 1989.

[5] E. A. Carter, R. G. Tompkins, E. Schiffrin, and J. F. Burke, "Cutaneous thermal injury alters macromolecular permeability of rat small intestine," Surgery, vol. 107, no. 3, pp. 335-341, 1990.

[6] E. A. Carter, J. N. Udall, S. E. Kirkham, and W. A. Walker, "Thermal injury and gastrointestinal function. I. Small intestinal nutrient absorption and DNA synthesis," Journal of Burn Care and Rehabilitation, vol. 7, no. 6, pp. 469-474, 1986.

[7] H. L. Walker and A. D. Mason Jr., "A standard animal burn," Journal of Trauma, vol. 8, no. 6, pp. 1049-1051, 1968.

[8] E. A. Carter, S. E. Kirkham, J. N. Udall, W. Jung, and W. A. Walker, "Thermal injury and gastrointestinal function. II. Evidence for the production of hepatic dysfunction in the rat following acute burn injury," Journal of Burn Care \& Rehabilitation, vol. 7, no. 6, pp. 475-478, 1986.

[9] P. F. Osgood, J. L. Murphy, D. B. Carr, and S. K. Szyfelbein, "Increases in plasma beta-endorphin and tail flick latency in the rat following burn injury," Life Sciences, vol. 40, no. 6, pp. 547554, 1987.

[10] R. S. Ward, R. P. Tuckett, K. B. English, and O. Johansson, "Cutaneous nerve distribution in adult rat hairy skin after thermal injury-an immunohistochemical study," Journal of Burn Care and Rehabilitation, vol. 19, no. 1, pp. 10-17, 1998.

[11] A. L. Warshaw, W. A. Walker, R. Cornell, and K. J. Isselbacher, "Small intestinal permeability to macromolecules. Transmission of horseradish peroxidase into mesenteric lymph and portal blood," Laboratory Investigation, vol. 25, no. 6, pp. 675-684, 1971.

[12] J. W. Alexander, C. K. Ogle, J. D. Stinnett, and B. G. Macmillan, "A sequential, prospective analysis of immunologic abnormalities and infection following severe thermal injury," Annals of Surgery, vol. 188, no. 6, pp. 809-816, 1978.

[13] D. E. Fry, T. W. Klamer, R. N. Garrison, and H. C. Polk Jr., "Atypical clostridial bacteremia," Surgery Gynecology and $\mathrm{Ob}$ stetrics, vol. 153, no. 1, pp. 28-30, 1981.

[14] R. N. Garrison, D. E. Fry, S. Berberich, and H. C. Polk Jr., "Enterococcal bacteremia: clinical implications and determinants of death," Annals of Surgery, vol. 196, no. 1, pp. 43-47, 1982.

[15] T. R. Ziegler, R. J. Smith, S. T. O’Dwyer, R. H. Demling, and D. W. Wilmore, "Increased intestinal permeability associated with infection in burn patients," Archives of Surgery, vol. 123, no. 11, pp. 1313-1319, 1988.

[16] W. G. Jones, J. P. Minei II, A. E. Barber et al., "Bacterial translocation and intestinal atrophy after thermal injury and burn wound sepsis," Annals of Surgery, vol. 211, no. 4, pp. 399-405, 1990.

[17] D. C. Morrison and J. L. Ryan, "Endotoxins and disease mechanisms," Annual Review of Medicine, vol. 38, pp. 417-432, 1987.

[18] Z.-I. Terashita, Y. Imura, K. Nishikawa, and S. Sumida, "Is platelet activating factor (PAF) a mediator of endotoxin shock?" European Journal of Pharmacology, vol. 109, no. 2, pp. 257-261, 1985.
[19] J. L. Wallace, G. Steel, B. J. R. Whittle, V. Lagente, and B. Vargaftig, "Evidence for platelet-activating factor as a mediator of endotoxin-induced gastrointestinal damage in the rat: effects of three platelet-activating factor antagonists," Gastroenterology, vol. 93, no. 4, pp. 765-773, 1987.

[20] D. A. Handley, J. C. Tomesch, and R. N. Saunders, "Inhibition of PAF-induced systemic responses in the rat, guinea pig, dog and primate by the receptor antagonist SRI 63-441," Thrombosis and Haemostasis, vol. 56, no. 1, pp. 40-44, 1986.

[21] J. L. Wallace and B. J. R. Whittle, "Prevention of endotoxininduced gastrointestinal damage by CV-3988, an antagonist of platelet-activating factor," European Journal of Pharmacology, vol. 124, no. 1-2, pp. 209-210, 1986.

[22] T. H. Wilson, Intestinal Absorption, W.B. Saunders Company, Philadelphia, Pa, USA, 1962. 

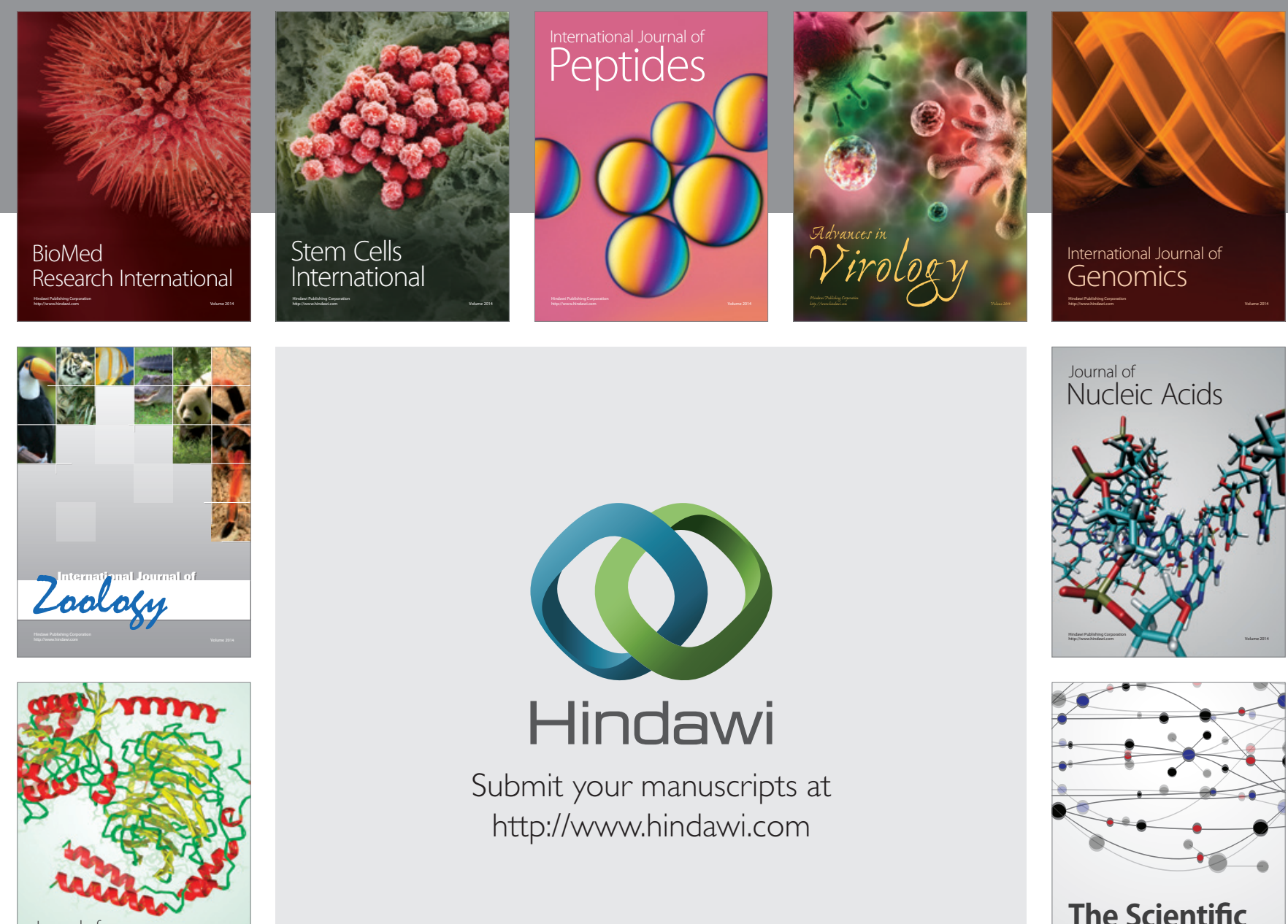

Submit your manuscripts at

http://www.hindawi.com

Journal of
Signal Transduction
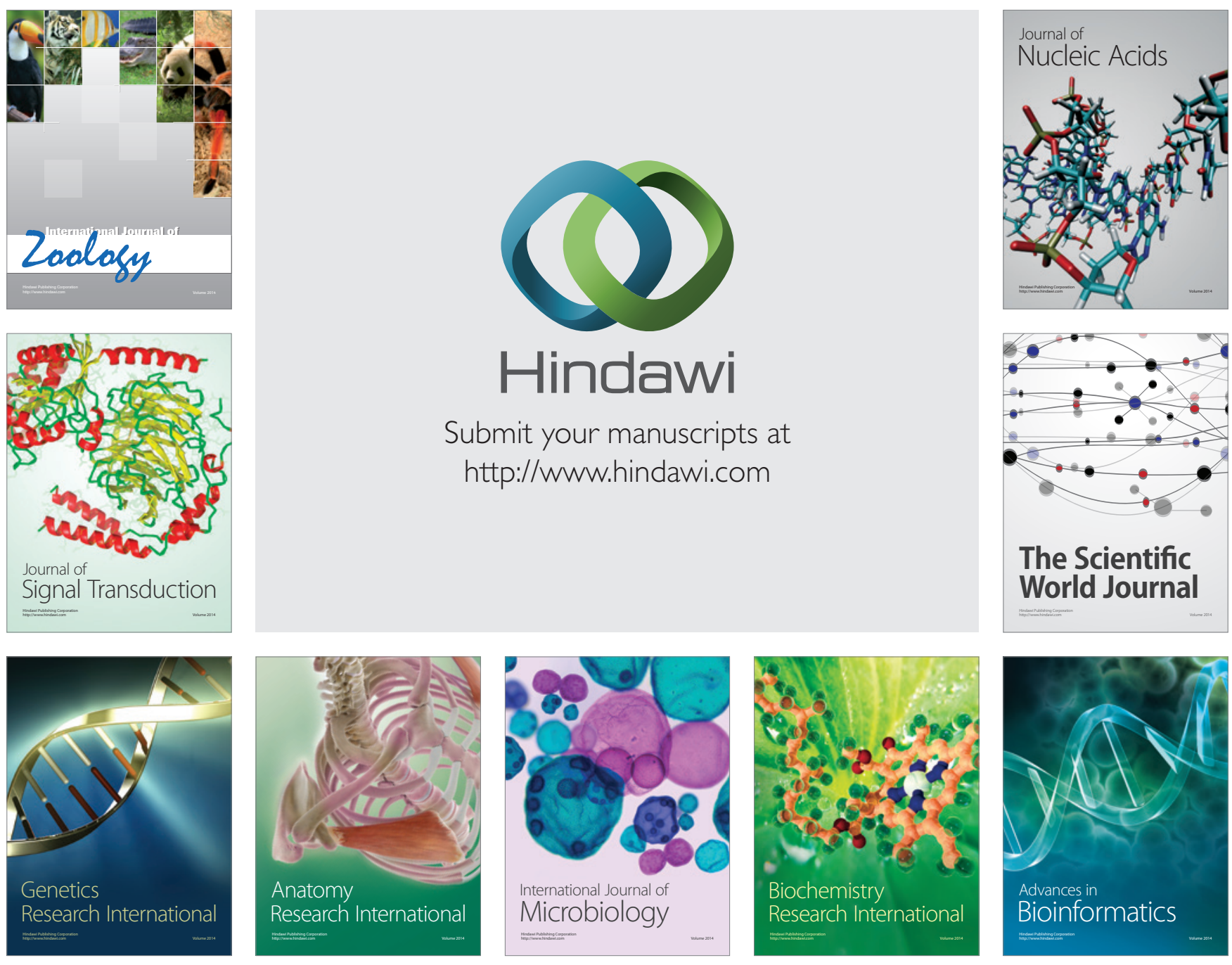

The Scientific World Journal
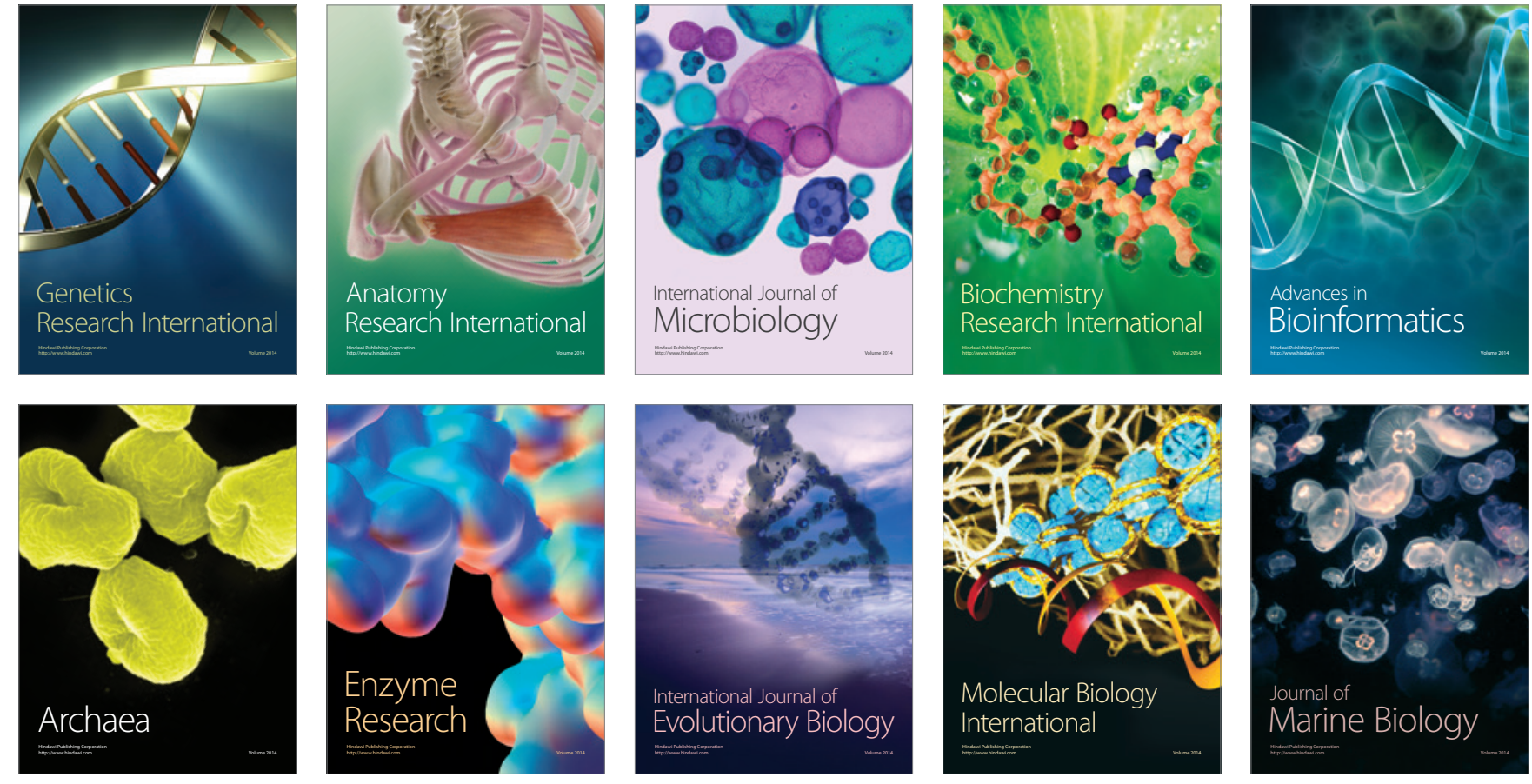